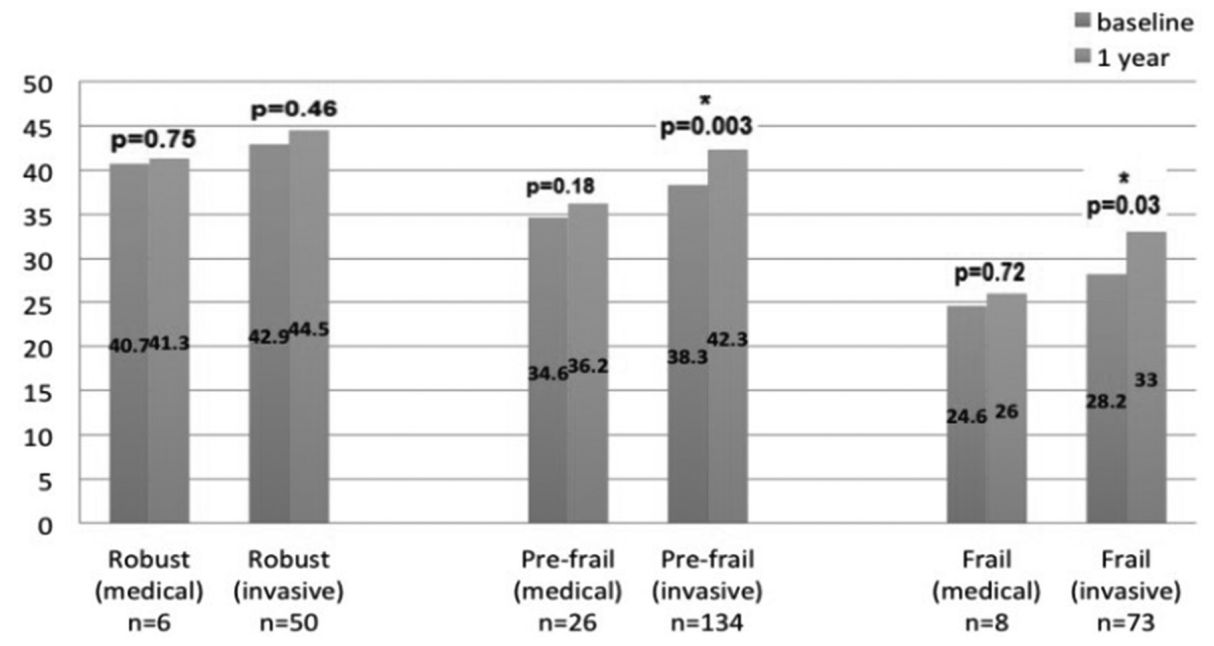

Abstract 165 Figure 1 Change in PCS by frailty and treatment

(NSTEACS) and is associated with a disproportionately high morbidity and mortality. We aimed to determine how frailty influences physical quality of life (QoL) outcomes after invasive treatment for NSTEACS.

Methods 298 patients aged 75 years with NSTEACS were enrolled into a multicentre prospective observational study, 297 (99.7\%) completed QoL questionnaires at baseline. Frailty was assessed using the Fried criteria, where a score of 0 is robust, 1 or 2 is pre-frail and 3 is frail. QoL was assessed by Short Form-36 (SF-36) questionnaire (license number QM033917) at baseline and 1 year follow-up. The normbased physical component score (PCS), an aggregated summary score of the 8 SF-36 subscales, is reported.

Results Mean age was $80.4 \pm 4.8$ years, $61.7 \%$ male, $86.6 \%$ received invasive treatment (percutaneous coronary intervention or coronary artery bypass grafting), and 234 (78.8\%) patients completed 1-year follow-up QoL assessment. At presentation, $56(18.9 \%)$ patients were robust, 160 (53.9\%) were pre-frail and $81(27.3 \%)$ were frail. There was no difference in proportional of patients received invasive management among frailty groups $(89.3 \%$ of robust, $83.8 \%$ of prefrail and $90.1 \%$ frail patients were treated invasively, $\mathrm{p}=0.30$ ). Increasing frailty was associated with decreased physical QoL at both baseline and 1 year $(p<0.001$ for both time points). Although all frailty groups saw an increase in mean PCS, this difference was only statistically significant in pre-frail and frail patients (robust: $42.7 \pm 10.7$ to $44.2 \pm 11.8$, $\mathrm{p}=0.409$; pre-frail: $37.7 \pm 11.1$ to $41.3 \pm 11.4, \mathrm{p}=0.001$; frail: $27.9 \pm 8.5$ to $32.5 \pm 12.6, \mathrm{p}=0.04)$. In addition, only pre-frail and frail patients who received invasive treatment saw this significant improvement in PCS between baseline and 1 year, although numbers receiving medical therapy only was low (figure 1).

Conclusion Although frail older patients with NSTEACS have a poorer physical QoL overall, our data suggest frailty is associated with a greater improvement from baseline QoL in those who receive invasive treatment.

Conflict of Interest None

\section{EVALUATION OF THE DIAGNOSTIC ACCURACY OF NURSE-LED ECG INTERPRETATION FOR A LARGE PRIMARY PERCUTANEOUS INTERVENTION SERVICE}

${ }^{1}$ Katie Linden*, ${ }^{2}$ Niall Herity, ${ }^{3}$ Mary McGeough. ${ }^{1}$ Belfast Health and Social Care Trust; ${ }^{2}$ Belfast Health and Social Care Trust; ${ }^{3}$ Belfast Health and Social Care Trust

\subsection{6/heartjnl-2019-BCS.163}

Introduction Primary PCI (PPCI) is the recommended treatment for patients presenting with ST-elevation myocardial infarction (STEMI). The catheterisation laboratory in the Royal Victoria Hospital in Belfast is activated when a diagnostic STEMI ECG is received from an ambulance crew or emergency department. The decision to activate is taken by CCU nurses. The aim of this study was to assess the numbers, sources, appropriateness and clinical outcomes of patients turned down for PPCI by this process and to assess the diagnostic accuracy of nurse-delivered ECG interpretation.

Methods The Belfast PPCI pathway is activated by trained nurses who act according to a regionally agreed protocol. Electrocardiograms (ECG) are transmitted by email or fax to a central hub and considered along with a focused telephone conversation. If the laboratory team is not activated, the referral is deemed a turndown. Clinical information and a record of the ECG is kept for each case and logged on a database. All turndowns between the beginning of April 2017 and the end of September 2017 were reviewed. A turndown was deemed inappropriate if retrospective review of the history and ECG demonstrated diagnostic features within PPCI pathway criteria.

Results Between 1st April 2017 and 30th September 2017 there were 957 referrals to the PPCI service. 595 (62.2\%) were turned down and in $362(37.8 \%)$ cases the laboratory team was activated. $15(1.6 \%)$ turndowns were deemed inappropriate of which 12 cases were due to inappropriate interpretation of the ECG and 3 cases were due to inaccurate interpretation of the clinical history. 51 cases were re-referred to the service a second time and of these 3 cases were 


Abstract 166 Table 1
\begin{tabular}{|c|c|c|c|}
\hline & $\begin{array}{c}\text { PPCl activation } \\
\text { criteria met }\end{array}$ & $\begin{array}{c}\text { PPCl activation } \\
\text { criteria not met }\end{array}$ & Total \\
\hline $\begin{array}{c}\text { Accepted for PPCl } \\
\text { PPCl }\end{array}$ & True positive 306 & False positive 56 & 362 \\
\hline Total & False negative 15 & True negative 580 & 595 \\
\hline Sensitivity & $95.33 \%$ & $\begin{array}{c}\text { Negative predictive } \\
\text { value }\end{array}$ & 957 \\
\hline Specificity & $91 / 19 \%$ & $\begin{array}{c}\text { Positive predictive } \\
\text { value }\end{array}$ & $84.48 \%$ \\
\hline
\end{tabular}

identified as having been inappropriately turndown on first referral. In 2014, the inappropriate turndown rate had been $3.1 \%$

$322(76.1 \%)$ patients in the turndown population required hospital admission and 290 (48.7\%) were admitted to a Cardiology service. The six-month mortality in the turndown population was $17 \%$ with a cardiac cause of death in $5.7 \%$ of cases. There were $2(13.3 \%)$ deaths in the inappropriate turndown group at six months, both due to cardiac causes.

The sensitivity, specificity positive and negative predictive values are shown in the Table. They indicate a high level of diagnostic accuracy for nurse-delivered ECG interpretation, and show that the system inclines towards greater sensitivity to miss as few patients as possible.

Conclusion In this PPCI service, the majority of patients referred are turned down appropriately. Clinical outcomes were similar among patients in the appropriate and inappropriate groups in the turndown cohort and the mortality rate in this group as a whole is relatively high. The rate of inappropriate turndown has decreased since 2014.

Conflict of Interest Nil

\section{CAN THE USE OF HIGHLY SENSITIVE TROPONIN ASSAY REDUCE THE LENGTH OF HOSPITAL ATTENDANCE AND IMPROVE PATIENT FLOW ON THE ACUTE MEDICAL UNIT?}

'Zoe Stone*, ${ }^{2}$ Elin Powell, ${ }^{3} J o h n$ Hounsell, ${ }^{4}$ Aaron Wong. ${ }^{1}$ Princess of Wales Hospital, Bridgend; ${ }^{2}$ Princess of Wales Hospital, Bridgend; ${ }^{3}$ Princess of Wales Hospital, Bridgend; ${ }^{4}$ Princess of Wales Hospital, Bridgend

\subsection{6/heartjnl-2019-BCS.164}

Introduction The 2015 European Society of Cardiology (ESC) guidelines for investigation and management of non-ST elevation myocardial infarction (NSTEMI) discuss a 'rule in/ rule out' $(0 \mathrm{~h} / \mathrm{h}$ ) algorithm for the use of one-hour highly sensitive troponin assay (figure 1) (1). Following the application of this algorithm to several large cohorts, the negative predictive value for NSTEMI in patients assigned 'rule out' exceeded 98\% according to these guidelines.

Aim The aim of this audit was to evaluate current practice of the assessment of cardiac sounding chest pain on the Acute Medical Unit (AMU) and to establish whether or not the introduction of highly sensitive one-hour troponin assay can shorten length of attendance and improve patient flow through the department.

Method Prospective data collection from October 2018 to December 2018. 99 patients presented to the AMU with cardiac sounding chest pain. Demographic data, ECG findings, troponin results, length of attendances and outcomes (admission, discharge and follow up) were captured.

Results 53\% were male. Mean age was 63.56 patients had initial troponin at presentation only. 12 patients had repeat troponin assays taken following 3 hours; 31 patients had repeat taken after 6 hours. Repeat blood tests for troponin were frequently taken at incorrect times. 9 patients had ischaemic changes on their initial ECG. Acute coronary syndrome was excluded in 88 patients; 26 patients were discharged with Emergency Access Cardiology clinic follow up for further investigation of chest pain. Of those patients who had repeat troponin tests, $77 \%$ of those could potentially have been discharged much sooner (avoiding some overnight stays) if the highly sensitive one-hour troponin assay was available (figure 2).

Conclusion This audit has highlighted several areas of disparity of standard practice in the assessment of chest pain on the AMU. We would like to undertake further work to improve standards. The recommendation of the one-hour troponin protocol suggested by ESC has the potential to significantly 